\title{
A Feasibility Study on a Cooperative Safety Application for Cyclists crossing Intersections
}

\author{
Daniel Thielen, Tobias Lorenz, \\ Marco Hannibal, Frank Köster, Jens Plättner \\ Institute of Transportation Systems \\ German Aerospace Center (DLR) \\ 38108 Braunschweig, Germany \\ Email:daniel.thielen@dlr.de, tobias.lorenz@dlr.de, \\ marco.hannibal@dlr.de, frank.koester@dlr.de,jens.plaettner@dlr.de
}

\begin{abstract}
Introduction of wireless vehicular communications enables a variety of new ITS use-cases [1, 2], allowing for cooperation between vehicles and infrastructure services. However the cooperation between vehicles and vulnerable road users (VRU) is not considered in these use cases. Currently there are already efforts to investigate the feasibility and the impact of systems to protect VRU [3-5]. Due to the continously increasing number of traffic participants in urban environments this paper focuses on protecting cyclists in intersection scenarios. Therefore this paper provides a feasibility study on a cooperative safety application for vehicles and bicycles using a vehicle equipped with Vehicle-to$X$ communication (ETSI ITS G5) technology and a commercial mobile device using consumer WLAN (IEEE 802.11g) at the bicycle. Within a demonstration it is proven that the set up and the deployment of such a system is feasible. Thus it could help to decrease the number of accidents between vehicles and bicycles or at least mitigate the impact of an accident.
\end{abstract}

\section{INTRODUCTION}

Development of future advanced driver assistance systems will most likely be driven by two key aspects: cooperation and automation. Cooperation means shared perception, decision making and action planning between several vehicles and traffic infrastructure (e.g. traffic light control) [1, 2]. From a technical viewpoint, this trend is supported by the introduction of communication (e.g. ETSI ITS G5) between vehicle and infrastructure or other vehicles (V2I, V2V or more general $\mathrm{V} 2 \mathrm{X}$ ). Using cooperative systems the overall driving efficiency and safety can be improved. Currently the research focus of cooperative assistance and automation systems (CAAS) comprises urban environments with a high degree of complexity caused by many different traffic participants reaching from cars, trucks and busses up to trams, cyclists and pedestriants as well as the complex urban infrastructure. Especially vulnerable road users (VRU) are endangered because in case of an accident their risk of heavy injuries or death is the highest. This aspect is reinforced by the electrification of vehicles and bicycles. In the latter case new electrical engines causes higher velocities for VRU. Additionally the future deployment of electric mobility in vehicles causes less noise emissions. Thus vehicles are more difficult to percept leading to a higher collision risk between VRU and vehicles. To tackle the challenge of better protecting VRU this paper presents a feasibility study on implementing a safety application to avoid or mitigate the impact of accidents between vehicles and cyclists as representatives of VRU. The presented study was carried out during the project venture CarS (Car-to-X Safety). To provide a deeper insight to the challenges to be tackled in this paper in Section II the motivation for investigating bicycles and vehicles as well as the chosen scenario are presented. Furthermore the challenges of cooperative awareness of both participants is outlined. In Section III the architecture of the whole system is described. An investigation on available sensors and data quality is performed and the implementation of the data exchange is depicted. Finally the concept of the algorithm for collision risk estimation is described. Subsequently an evaluation of the safety application within the test scenario is performed. The paper ends up with a summary of the presented work and a conclusion is drawn.

\section{A. Related Work}

Considering the current publications in the area of cooperative systems comprising VRU there are several existing projects and studies. On the European level the currently running project WATCH-OVER [5] is a representative dealing with VRU and vehicles. There eight traffic scenarios relevant for this configuration were identified using user surveys. The aim is to increase the safety of VRU using advanced driver assistance systems (ADAS). Thereby the used sensors are invehicle sensors like cameras. The collision risk estimation is done only in the vehicles. However for the warning concept the vehicle driver and the cyclist shall be warned. Therefore data exchange is realized using wireless communication technologies like WLAN, ZigBee or Ultra-Wide-Band (UWB). Another project started in 2011 is the Dutch project InnoCross [3]. One goal of this project is to decrease the number of accidents with cyclists by decreasing the number of red light violences of cyclists by implementing a green light assistant. Furthermore a collision warning system between vehicles and cyclists is implemented too. This project mainly focuses on a centralized detection and warning concept in dangerous traffic situations. Thereby an ITS Roadside Station (IRS) detects and assesses the risk of a traffic situation using e.g. cameras. 
If there is a dangerous situation the vehicle and the cyclist are warned using ETSI ITS G5 for the vehicle and UMTS or LTE for the mobile device of the cyclist. The pre-FOT SaveCap [4] is a Dutch sensor field test where five vehicles of the dutch telecom are equipped with multiple sensors. These vehicles gather information about behaviour of VRU in real traffic and characteristical values to distinguish dangerous and non-dangerous traffic situations between vehicles and VRU with the aim to decrease the number of false alarms in future ADAS. The german project AMULETT ${ }^{1}$ also aims to protect VRU. However there RFID technology is used to detect VRU not visible for the vehicle driver.

\section{Problem Statement}

\section{A. Introduction}

Looking at accident statistics it is proven that an important amount of fatal and non-fatal accidents occur at intersections. In [3] it is outlined that in the EU-13 5,476 casualties or $21.3 \%$ of all traffic incidents occur at intersections. Furthermore it is outlined that junctions are responsible for $30 \%$ to $60 \%$ of wounded road user and $16 \%$ to $36 \%$ of fatal incidents within Europe. The database of these values are measurements in France, Great Britain and Germany. Considering all European capitals the mean death rate for cyclists as one group of traffic participants is about $5 \%$ to $10 \%$ of all traffic death (cmp. [3]). However there are significant regional differences caused by the different numbers of cyclists in the cities. In cities like e.g. Amsterdam, Berlin and Copenhagen this value is between $10 \%$ to $30 \%$. Results from studies of the German Federal Statistical Office in 2006 showed that in 2005 there were 78,434 accidents where cyclists were involved. Compared to former studies this is an increasement of $6.5 \%$. Furthermore the percentage of fatal cyclist accidents increased by $21 \%$. The most dangerous scenario identified for accidents between cyclists and vehicles is the cross traffic scenario which referring to the GIDAS ${ }^{2}$ database of VW causes $70 \%$ of all accidents. The potential danger of this scenario is reinforced by possible obstacles blocking the line of sight between cyclists and vehicles, by the higher velocities of electric bicycles, by the low noise emission of future electric vehicles and by cyclist driving the cycleway in the wrong direction. Within this paper the cross traffic scenario is investigated in detail and a feasibility study on a safety application to protect cyclists using a consumer mobile device with WLAN $(802.11 \mathrm{~g})$ and a vehicle equipped with Vehicleto-X communication technology.

\section{B. Scenario}

In Figure 1 the basic scenario identified in the previous subsection is outlined. The left figure shows an accident situation between a vehicle and cyclist within the basic scenario. Considering the right figure a schematic approach of solving the dangerous situation using the communication between the

\footnotetext{
${ }^{1} \mathrm{http}: / /$ www.projekt-amulett.de/

${ }^{2}$ German In-Depth Accident Study
}
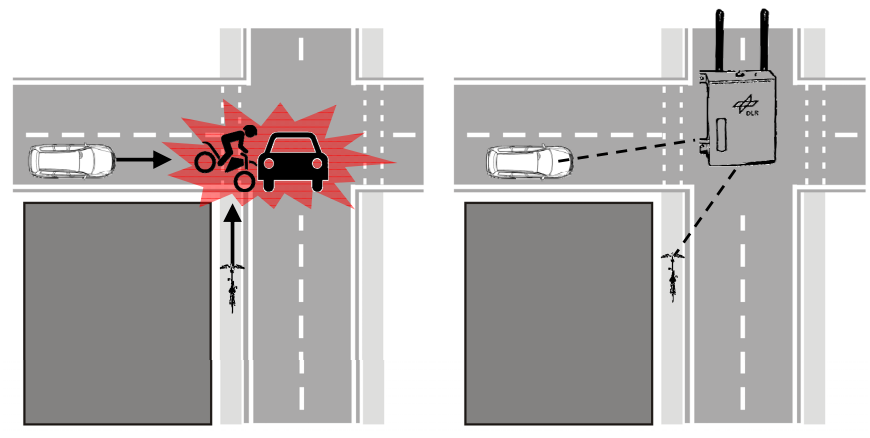

Figure 1. Basic scenario of the feasibility study

vehicle and the cyclist using an ITS Roadside Station (IRS) as bridge between WLAN 802.11g and V2X technology (ETSI ITS G5) is depicted. Because the line of sight is blocked between both traffic participants classical in-vehicle sensors like radar, cameras, laser scanner, etc. cannot be used to detect the situation. However as outlined in Subsection I-A there are further possibilities to do so. Within this paper motivated by the content of the project venture CarS it was decided to use a wireless communication link between the bicycle and the vehicle. The used sensors in the vehicle are e.g. velocity sensor, GPS receiver, pedal position sensor etc. The sensors of the mobile device like GPS receiver, accelerometer etc. are used as data source for the bicycle. The sensors used in this paper are investigated in the following sections. The aim is to warn the vehicle driver based on the state information of his own vehicle and the state information of the bicycle about a possible collision risk with the cyclist. Therefore the warning has to be in time, reliable and the warning must only occur in real dangerous situations.

\section{Proposed Solution}

Subsequently an architectural and technical solution is proposed to fulfill the needs of the described scenario successfully. First a system architecture depicting the single components of the system as well as the communication links between these components are presented. Afterwards details on every single component Mobile Device, Vehicle and Collision EstimationConcept are outlined.

\section{A. System Architecture}

In Figure 2 the system architecture of the whole cooperative safety application is presented. The main components of this architecture are (start in the right corner going clockwise) the Mobile Device, the IRS and the Vehicle.

For the Mobile Device the available communication technologies are outlined. These are WLAN $(802.11 \mathrm{~b} / \mathrm{g} / \mathrm{n})$, Bluetooth and cellular communication. Within this feasibility study WLAN is chosen to exchange data between the Mobile Device and the IRS. Furthermore the build-in sensors that are used during the study to determine the state, position and heading of the bicycle are presented. Subsequently the data quality of these sensors is considered in more detail. The Mobile 


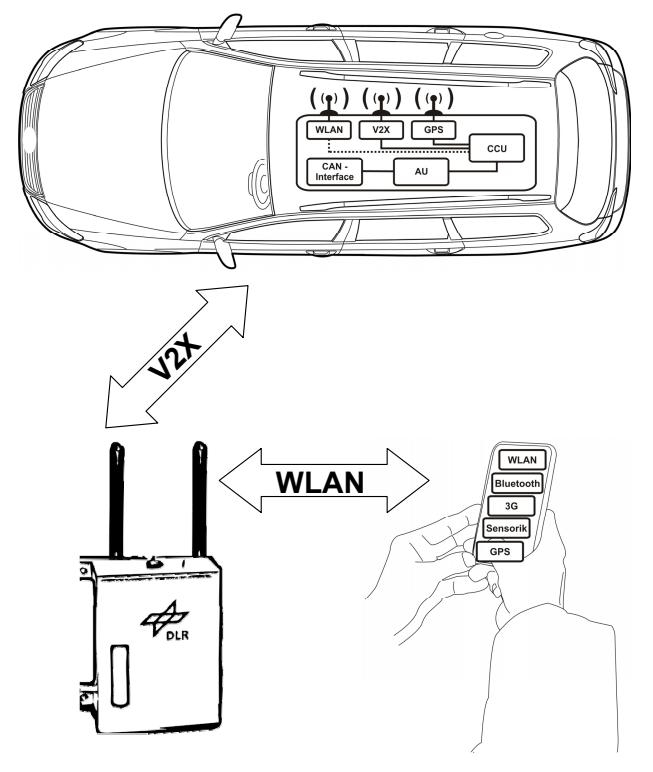

Figure 2. Architecture of the whole System

Device that is used (Samsung Galaxy SII) has a translational acceleration sensor, a gyroscope and a GPS receiver. The state information of the bicycle is transmitted to the IRS via consumer WLAN using a Cooperative Awareness Message (CAM) defined in the Car2Car Demo 2008 in Dudenhofen [6]. At the IRS the CAM is received and forwarded to the Vehicle using ETSI ITS G5. Therefore the IRS is build up with a Communication and Control Unit (CCU) and an Application Unit (AU) (cmp. [7]). As CCU for ETSI ITS G5 a Linkbird V3 from NEC is used and for the consumer WLAN communication a PCMCIA Ubiquiti WLAN card with an Atheros Chipset is used in master mode. The AU is a computer usable in the automotive domain running a Linux OS. In the Vehicle a Linkbird V3 from NEC as CCU and an $\mathrm{AU}$ is running too. At the $\mathrm{AU}$ in the vehicle the state information of the bicycle is received and assessed via the CAM. Additionally the state information of the Vehicle gathered from the CAN-Interface and the Antaris 5 GPS receiver are received and assessed. Using the Collision Estimationalgorithm an collision risk assessment of the current situation is executed and if necessary the vehicle driver is warned using acoustical and visual feedback.

\section{B. Mobile Device}

1) Introduction: In regard to a fast software development for mobile devices the Android platform was selected to realize the mobile application. The availability of a rich set of tools (e.g. OS, middleware, development tools) including an extensive documentation to nearly all featured properties of the operating system and a variety of supported hardware emphasised this selection. An appropriated mobile device related to the outlined Android operating system is the Samsung Galaxy SII. It bases on Samsungs self-developed 32-bit RISC processor System on a chip (SoC) Exynos 4210. This SoC

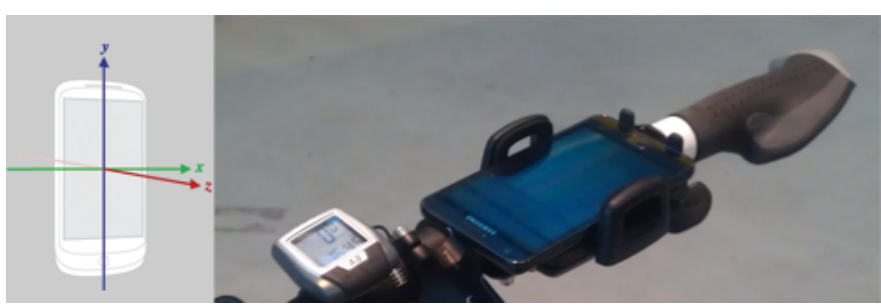

Figure 3. Definition of the coordinate system used by the SensorEvent API (left) and mounted Samsung Galaxy SII on bicycle handlebar (right)
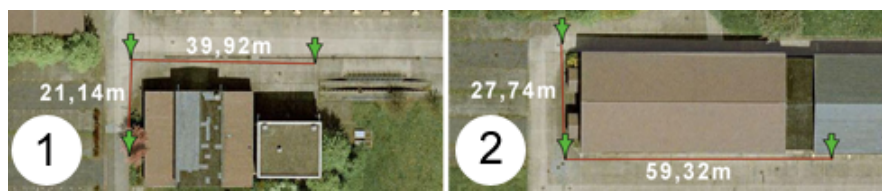

Figure 4. GPS test scenarios with marked checkpoints for GPS-data-logging (green arrows) on a former military base in the south-east of Brunswick, Lower Saxony, Germany; Source: Google maps

integrates different components and enables the usage of a wide range of sensors.

2) Sensors: The devices used is equipped with GPS, furthermore a gyroscope, a accelerometer and other sensors which are not considered in this context. The version of the installed Android operation system was Gingerbread version 2.3.3.

GPS Sensor: Due to the scenario the usage of the GPS is essential. Therfore different GPS accuracy tests were performed. Considering real conditions of urban scenarios these tests took place at the begin of June 2011 at DLR's test field, a former military base in the south-east of Brunswick, Lower Saxony, Germany. For these tests a bike equipped with a Samsungs Galaxy SII was used. In this device a Broadcom BCM4751 GPS-Sensor is assembled.

Related to the definition of Androids coordinate system used by the SensorEvent API (see figure 3) the Y axis of the device was mounted parallel to horizontal and the $\mathrm{Z}$ axis pointed up. During the test execution a small application installed on the device logged the measured GPS position, speed and accurancy.

The GPS accuracy was investigated during 19 test runs in two different scenarios (see figure 4). The GPS positions were logged at the starting-, turning- and endpoint manually. In preparation of this evaluation these three points were determined by a high precision differential GPS-System Topcon GRS-1 as a reliable reference for the data interpretation. This handheld GPS-receiver obtains a real time kinematic (RTK) accuracy of $1 \mathrm{~cm}$ (horizontal) and $1.5 \mathrm{~cm}$ (vertical) [8]. Due to realistic urban conditions the driven course was intentionally planned close to buildings (height of $5 \mathrm{~m}$ ).

Accelerometer- and Gyroscope-Sensor: In addition to the GPS sensor, the mentioned acceleration sensor and gyroscope were considered. Both sensors are ST Microelectronics components, type K3DH (accelerometer) and K3G (gyroscope). In order to enrich the GPS-data and to detect a change in the cyclist 


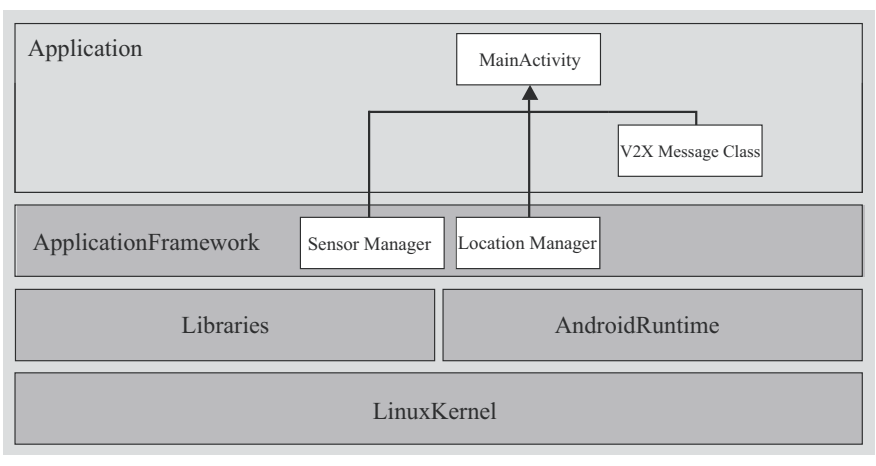

Figure 5. Android Application - Architecture for the described scenario

behaviour, the accelerometer and gyroscope values were also investigated in further simple test drives. The underlying scenario for both sensors was a $90 \mathrm{~m}$ course, straight for accelerometer measurements (including an emergency brake at course end) and with a 90 degrees left turn at halfway for the gyroscope. The main objectives of these tests were to measure changes of the bicycle speed or driving direction. Therefore the above mentioned bike with the mounted Samsung Galaxy SII was used. A small Android application installed on the mobile device logged the sensors values.

3) Implementation measurement application: The general architecture design of the implemented measurements applications is presented in Figure 5. The software was used on the mentioned device for all sensor measurements (GPS, accelerometer and gyroscope). In the main activity (an activity represents a single screen with a user interface) the application registrates a LocationListener and a SensorListener for receiving notifications from the SensorManager (responsible for accelerometer and gyroscope) or LocationManager (GPS) when sensor values from the mobile device have changed.

Measurement excecution: In preparation of each test drive the application had to be started on the Samsung device. After that the ciclyst had to activate data logging before he started driving. During each drive the data of the accelerometer and gyroscope were logged automatically every $250 \mathrm{~ms}$. In contrast to this the record of the GPS data had to be initiated manually by the user clicking a button when he passed one of the checkpoints (each checkpoint is indicated as green arrow in figure 4).

4) Implementation assistance application: The underlying software design for the assistance application corresponds to the above mentioned measurement application (see figure 5). This also applies for the listeners registration to receive sensor values. In order to enable the assistance system it is neccessary to open the application and keep it running.

In contrast to the measurement application this software did not store its data localy. In contrast it uses the V2X Message Class, an JAVA-implementation of a Cooperative Awareness Message (CAM) defined in the Car2Car Demo 2008 in Dudenhofen [6] to broadcast its sensor data using WLAN 802.11b every $250 \mathrm{~ms}$.

\section{Vehicle}

1) Introduction: The base vehicle used for the scenario, FASCar, is a 2009 Volkswagen Passat Variant which is heavily modified from series-production status in order to serve as a research platform for different purposes. Among these modifications, an electronic interface that allows the automation to control lateral and longitudinal driving dynamics was added and the vehicle is equipped with different sensors. Furthermore a HMI display (1280x480) is installed to be able to investigate various HMI concepts.

2) Sensors: Besides the actuators mentioned in last subsection the test vehicle FASCar has been equipped with several sensors for the task of positioning and environment perception. For the positioning system a commercial system based on differential GPS fused with an inertial system (INS) is applied. Furthermore a commercial u-blox Antaris 5 GPS receiver is mounted at the FASCar. For environment detection, several sensors have been installed, such as laser scanners, cameras and radar. Additionally the data of sensors from series-production are available via CAN interface e.g. velocity, light, rain sensor, etc. Within the framework of this paper mainly the data of Antaris 5 GPS Receiver, the brake pedal position and the velocity information via CAN-Interface is important.

3) Implementation: For the implementation of the necessary concept the DLR's self developed middleware DOMIN$I O N$ is used $[9,10]$. DOMINION is inspired by the idea of service oriented architecture. Following the concept of service orientation, basic services like hardware access are encapsulated in reusable and loosely coupled services. For any use-case (research facility) a certain set of basic services is provided for flexible orchestration of new assistance and automation functions. Furthermore DOMINION also features monitoring and logging of data for fast assessment of experiment data.

Thus some already available DOMINION services could be used to run the presented ADAS like the service receiving the GPS information of the vehicle and the service gathering the information from the vehicle CAN. However it was necessary to to implement a service to collect, to process and to manage the state information of the vehicle and the bicycle for there use in the Collision Estimation Algorithm. This algorithm is represented by another DOMINION service implemented during the project. The concept underlying this algorithm is presented in Subsection III-E. All these services are implemented in $\mathrm{C} / \mathrm{C}++$ running on an Ubuntu linux system with a real time kernel extension and exchange their data using DOMINION's shared memory concept.

\section{Sensors and Data Quality}

For the implementation of the safety application the following sensors are investigated related to their data quantity and quality. These sensors are the acceleration sensor, the gyroscope and the GPS sensor of the Mobile Device as well as the velocity sensor and GPS sensor of the Vehicle. 

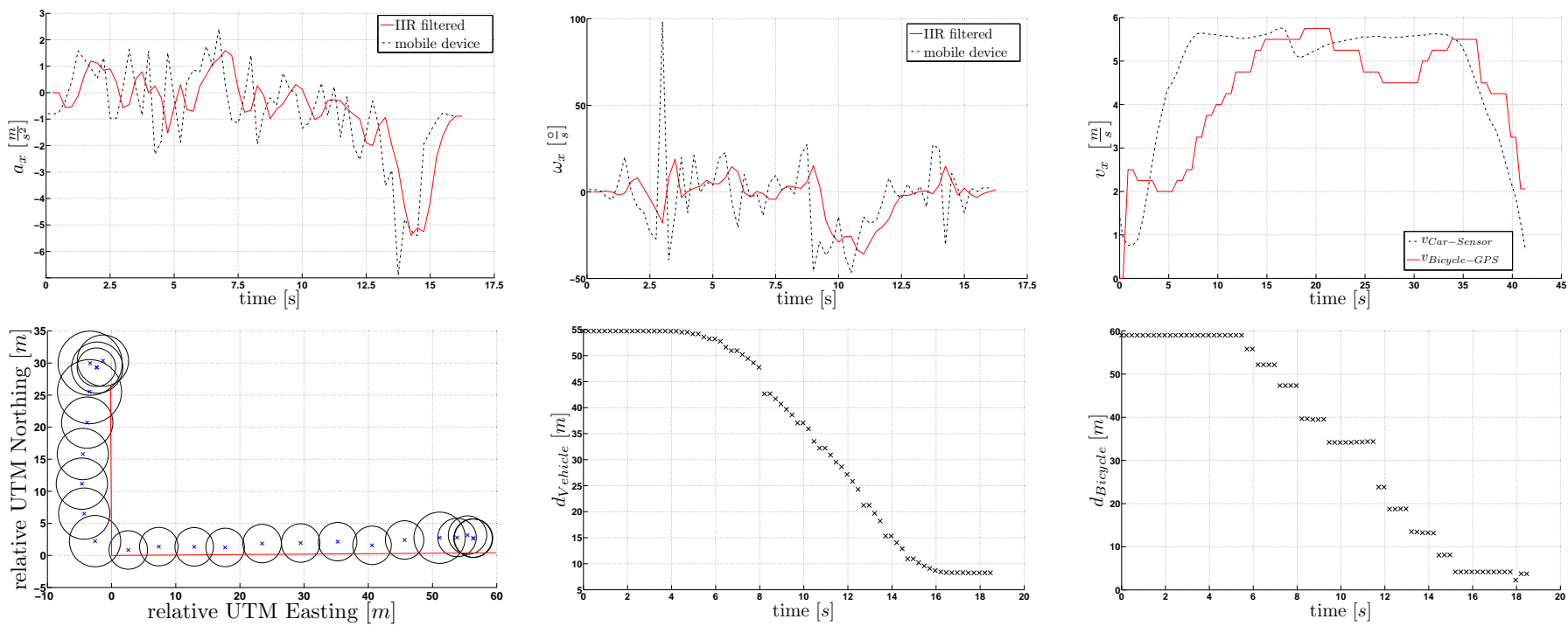

Figure 6. Sensor data plots

1) GPS Sensor (Mobile Device): As mentioned in section III-B a test drive was performed to investigate the Samsung Galaxy SII sensor quality. In figure 6 (second row, left chart) the GPS sensor related results of one drive have been plotted. The straight lines represent relatively the course of testscenario 2 between starting-, turning- and end-point (see figure 4).

Additionally the logged mobile device GPS-coordinates are displayed also relatively to the course distances as crosses supplemented by their predicted accurancy deviation represented as circles. Both values are accessible by the Android API.

As a result deviations between high accurancy course (red line) and logged mobile device GPS positions (crosses) are visible and mostly placed within the Android-predicted deviation radius. Another outcome of these test drives were the determination of a 95 percent accuracy $^{3}$ for the three GPScheckpoints:

- horizontal accuracy of $<5.73 \mathrm{~m}$

- vertical accuracy of $<9.66 \mathrm{~m}$

- 2D accuracy of $<9.73 \mathrm{~m}$

The average 2D deviation for all test drives and checkpoints amounts to $4.63 \mathrm{~m}$. As compared to the official U.S. government Global Positioning System Standard Positioning Service Performance Standard (GPS SPS PS) the minimum level of Singal-In-Space range accuracy of $7.8 \mathrm{~m}$ is mentioned [11]. Furthermore a particular system behavior was detected during the data evaluation: the GPS update rate of the mobile device amounts to approximately one second. Regarding Samsungs specification for the Galaxy SII as well as Androids operation system description for version 2.3.3 it was not possible to find out what the minimal time interval for GPS notifications could be. Thus, the implemented tool for looging GPS-data used

\footnotetext{
3 a circle's radius, centered at the determined TOPCON position, containing 95 percent of the points in the horizontal scatter plot
}

the lowest parameter admitted by the Android API to obtain notifications as frequently as possible.[12] This behaviour is also visible in figure 6 (lower row, right chart). This chart is the result of a simple GPS data investigation. On a straight course of $60 \mathrm{~m}$ the GPS data was captured in a frequency of $250 \mathrm{~ms}$. This plot approves the mentioned sample-rate estimation of approximately one second.

Concerning the velocity from GPS sensor figure 6 (first row, right chart) the above mentioned update rate of one second becomes visible. The red line depicts the bicycle and thus the GPS speed from Samsungs mobile device. It represents a delayed and gradually chart instead of the smooth outline of the vehicular CAN-data (black dashed line).

2) Accelerometer (Mobile Device): In addition to the GPS sensor the accelerometer also provides the acceleration of the mobile device but in a higher frequency. Due to the unavailability of the sensor specifiation from the manufacturer ST Microelectronics, DLR performed own measurements which indicated a sample rate less than $100 \mathrm{~ms}$.

The acceleration sensor data as well as the gyroscope were investigated to possibly use them for further improvements of the recorded GPS data.

In figure 6 (first row, left chart) the accelerometer sensor related results of one drive have been plotted. The raw data is depicted in a black dashed line. Furthermore the red line outlines the data after a infinite impulse response filter (IIR) was applied. The data was recorded in a sample rate of $250 \mathrm{~ms}$. The chart clearly shows the excecuted emergency brake during the test drive, apperently as an acceleration value of approximately $-5 \frac{\mathrm{m}}{\mathrm{s}^{2}}$ (filtered) and $-7 \frac{\mathrm{m}}{\mathrm{s}^{2}}$ (raw).

3) Gyroscope (Mobile Device): As mentioned in the section before own measurements were performed to determine the sensors sample rate. The results for the gyroscopes were determined less than $10 \mathrm{~ms}$. 


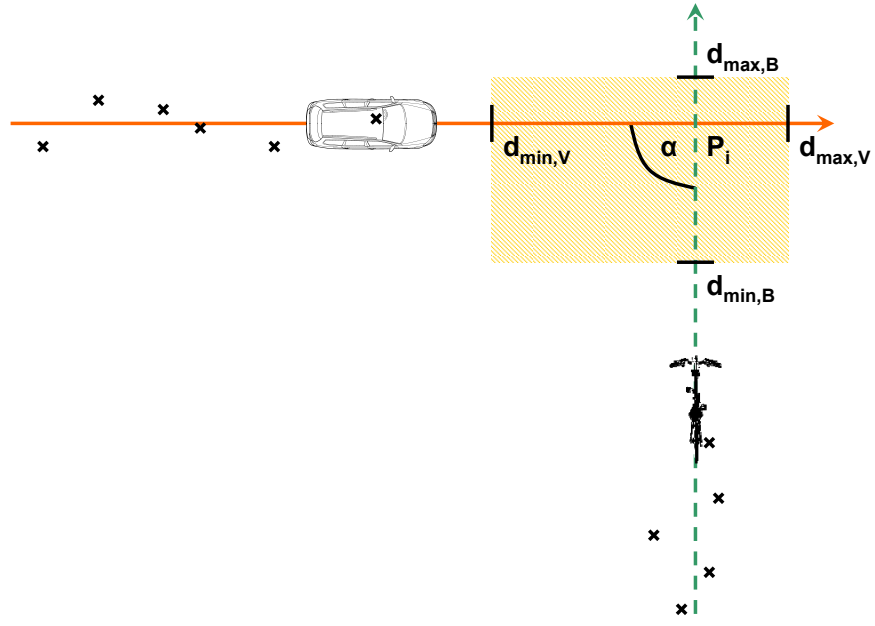

Figure 7. Schema of the collision risk estimation

In figure 6 (first row, center chart) the gyroscope sensor related results of one drive have been plotted. Respectively to the accelerometer chart, the gyroscope data was also recorded in a frequency of $250 \mathrm{~ms}$ and plotted as raw results (blackdashed line) and IIR filtered results (red line). Indeed the 90 degrees left turn at halfway during the test drive is visible. As compared to the accelerometers chart, the result is not so clear but noticeable. It can be assumed that an interpretation of a dataplot with various driving maneuvers might be difficult.

4) GPS Sensor (Vehicle): In the centered plot of the lower row of Figure 6 the sensor results from the Vehicle GPS sensor are presented. On a straight course of $55 \mathrm{~m}$ the GPS data was captured in a frequency of $250 \mathrm{~ms}$. The scenario of this data set was captured by driving parallel to a small but long building in a distance of approximately $15 \mathrm{~m}$. The sample rate of the Antaris 5 GPS receiver is $250 \mathrm{~ms}$. Looking at the plot small discontinuities are visible. These discontinuties are in a range of approximately $\pm 5 \mathrm{~m}$.

\section{E. Collision Estimation - Concept}

Considering the sensor data results of the Mobile Device and of the Vehicle the following data is chosen to be the input data of the collision estimation algorithm (CEA).

- Vehicle \& Mobile Device

- UTM Northing $(y)$ \& UTM Easting $(x)$

- GPS - Heading $(\phi)$

- Vehicle

- Brake pedal position

- Velocity from CAN-data $\left(v_{V e h i c l e}\right)$

- Mobile Device

- Velocity from GPS $\left(v_{\text {Bicycle }}\right)$

- Angular velocity $\left(\omega_{z}\right)$

- Longitudinal acceleration $\left(a_{x}\right)$

The output data of the CEA is chosen to be the time to collision (ttc) of the identified collision objects and the direction the objects are coming from (left or right). The mathematical concept underlying the CEA is depecited in Figure 7. The starting point is the collection of the state information of the last 10 valid input tuples for the vehicle and the bicycle. A new received data tuple is considered as valid if the difference between timestamp of data creation and the current timestamp is not larger than a certain value (max. $2 \mathrm{~s}$ ), if all values of the data tuple are within their predetermined range and if the information of the incoming data differs from the already available information (state of the system has changed). Based on this information a calculation of the intersection point as the position where the collision between the objects will take place is performed. To predict the movement of both objects a linear estimation is performed using the least square method (LSM). Using these method at least three valid tuple of position data are necessary to perform the LSM. In the equations 1 and 2 the set up of the matrices and the calculation of the LSM is presented.

$$
\begin{aligned}
& \underbrace{\left[\begin{array}{l}
y_{1} \\
\cdots \\
y_{n}
\end{array}\right]}_{\underline{y}}=\underbrace{\left[\begin{array}{cc}
x_{1} & 1 \\
\ldots & \ldots \\
x_{n} & 1
\end{array}\right]}_{\underline{M}} \cdot \underbrace{\left[\begin{array}{l}
p_{1} \\
p_{2}
\end{array}\right]}_{\underline{p}} \\
& \underline{p}=\left(\underline{M}^{T} \underline{M}\right)^{-1} \underline{M}^{T} \underline{y}
\end{aligned}
$$

The result of the LSM method calculation are the parameters of two linear functions describing the direction of the movement of the bicycle and the vehicle. Based on this linear functions an intersection point calculation is performed. If the intersection point calculation is performed successfully a safety area is build up around the intersection point (cmp. Figure 7). There $d_{\min , V}$ and $d_{\max , V}$ are the distances from the intersection point along the linear function of the vehicle in driving direction. For the bicycle this procedure is performed in a similar way using $d_{\min , B}$ and $d_{\max , B}$. However these distances can be parameterized online for example if the GPS accuracy changes. In the next step the position information where the vehicle and the bicycle enters and leaves the safety area are used to calculate the times of entering and leaving the safety area for the vehicle and the bicycle. Therefore a movement with a constant velocity taken from the last valid data tuple is used. Thus two time intervals one when the bicycle will be in the safety area and one when the vehicle will be in the safety are available. If these two intervals overlap there is a risk of collision and the time-to-collision (ttc) is derived. Now the direction the collision object (bicycle) is coming from $(\alpha)$ is calculated using the heading taken from the last valid data tuple.

However the CEA has some limitations. As outlined in former sections only cross traffic scenarios are considered. Thus the algorithm is not working if a collision object is coming from ahead or from behind $\left(45^{\circ} \leq|\alpha| \leq 135^{\circ}\right)$. There no calculation of collision risk will be performed. The reason for this is that the accuracy of the data is too low to be able estimate whether the bicycle is a danger for the vehicle or not. Furthermore if the bicycle is coming from ahead or from 


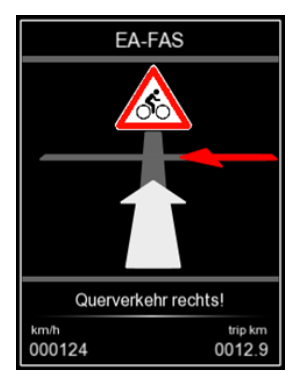

Figure 8. Pictogram for collision danger from the right

behind the vehicle driver is able to percept it by his own. Another limitation of the CEA is the number of regression points used for the LSM. If more regression points are used for the LSM the accuracy of the prediction horizon for a straight movement can be increased because more values are available to set up the linear regression function. However too many regression points lead to the case that a turning maneuver especially of the bicycle cannot be determined in time. To avoid this the accelerometer and the gyroscope data of the bicycle are used to detect braking and cornering maneuvers of the cyclist in time because the update rate of this information is $250 \mathrm{~ms}$ where the GPS update rate of the bicycle is 1000 ms. In the former section the feasibility of detecting such maneuvers using the sensor of the mobile device is shown. Finally a collision risk warning is only be provided if the vehicle driver does not brake, if the cyclist is not braking or cornering and if both traffic participants are faster than $2 \frac{\mathrm{m}}{\mathrm{s}}$.

\section{F. HMI - Concept}

For the HMI concept it was decided to only warn the vehicle driver first. Therefore an acoustical warning signal followed by a pictogram provided in the middle of the digital instrument cluster is chosen. In Figure 8 the pictogram shows the warning for a potential danger of collision coming from the right side. To be able to depict this flexible HMI the digital instrument cluster within the FASCar $(1280 x 480)$ is used. For the implementation of this cluster the software ScadeDisplay and ScadeSuite from Esterel was used. With this software the creation of graphical elements within ScadeDisplay is easily possible. These elements can afterwards be linked with state knowledge using ScadeSuite. Finally plain C-code is automatically generated by the software that is subsequently integrated into DOMINION (cmp. [13]).

\section{EvaluATiON}

In this section the system for avoiding or mitigating the impact of cross traffic collision between cyclists and vehicles is evaluated. Therefore a scenario similar to the left plot in Figure 4 is set up. The line of sight between the cyclist and the vehicle driver is fully blocked. Thereby the cyclist is driving southern direction along the building where the vehicle is driving in eastern direction. In Figure 9 the results of these trials are presented. In the left plot of Figure 9 the GPS data relative to the vehicle's starting point is presented for the bicycle and the vehicle. Furthermore the calculated intersection points are presented. It is visible that the intersection points are within a very small area of approx. $5 \mathrm{~m}$ x $5 \mathrm{~m}$ with one value being at the outer edge of this area. However this accuracy is sufficient to estimate a collision risk. Considering the right figure the $t t c$, the velocites of both traffic participants and the direction the collision object is coming from is depicted. The vehicle is driving at a velocity of about $6 \frac{\mathrm{m}}{\mathrm{s}}$ while the bicycle is driving $4 \frac{\mathrm{m}}{\mathrm{s}}$. If $t$ tc drops below the warning limit (in this case $5 \mathrm{~s}$ ) the warning is indicated and the acoustical and visual warning is presented. In this case the cyclist is coming from the left side indicated by the direction value ' 4 '. However the plots of the $t t c$ and object direction start providing data larger than zero if the ttc limit condition and the conditions mentioned in the former section as limitations of the CEA are fulfilled. In the moment the vehicle driver starts braking (approx. at $11.7 \mathrm{~s}$ ) the CEA algorithm stops providing data about the collision risk because one of the conditions is not fulfilled. These evaluation trials were executed several times with different limits for the $t t c$ triggering the warning as well as for different sizes of the safety area. Finally it could be shown that a reproducibility of the presented results is possible. However the warning limits of the $t t c$ and especially the sizes of the safety area heavily depend on the accuracy of the GPS values. When the GPS accuracy is low the safety area have to be enlarged to obtain a reliable warning. Thus it was difficult to find a setup working reliable at different places e.g. open area vs. city vs. alley. Of course the safety area can be chosen very large (e.g. $10 \mathrm{~m}$ $\mathrm{x} 10 \mathrm{~m}$ ) to serve the needs for all these scenarios but the moments the warning occurs will differ although the ttc limit remains constant. Furthermore the scenarios where the system must not warn the vehicle driver were tested too. Considering these results there was no false alarm for a cyclist coming from ahead or from behind. What was much more difficult to predict is the estimation in case the cyclist is crossing in a short distance in front of the vehicle. There a high accuracy of GPS values is needed to not warn the driver in these situations. With a low accuracy of GPS values and thus a larger safety area this situation was not detectable accurate. Respectively the mentioned consideration of accelerometer and gyroscope with the intention of detecting driver-behavior might be a possibility for further investigations. However in situations where one of the both traffic participants brake before the occurance of the warning signal no warning occurs in all tested situations.

\section{Summary \& CONClusion}

Within this paper a feasibility study on setting up a collision warning system based on wireless communication between a commercial mobile device, an IRS and a vehicle equipped with V2X technology is presented. It was shown which sensor accuracy is possible using the mobile device to determine the current state of the bicycle movement. Additionally the same was done for the sensors mounted in the vehicle. Based on that experiences an algorithm to estimate the collision risk was presented and evaluated. Summarizing the results it can be said that the setup of the intended collision warning 

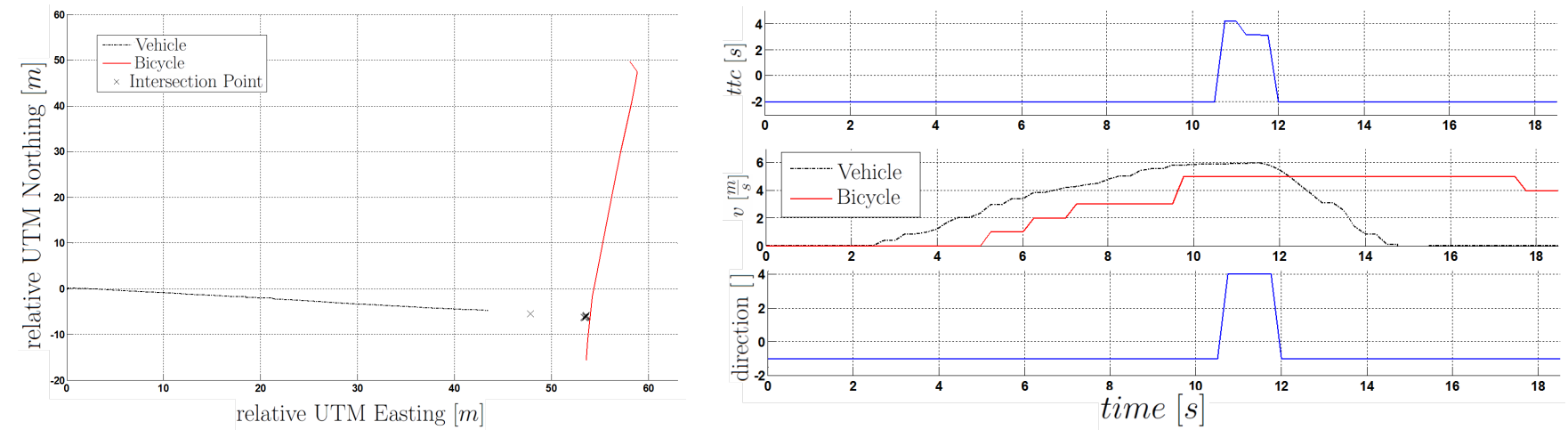

Figure 9. ADAS evaluation plots

system is feasible. However for the future the accuracy of the positioning information especially for the mobile device has to be improved. Furthermore the update rate of state information of the mobile device should be higher because an update of $1 \mathrm{~s}$ is very low. Finally an investigation of an additional warning mechanism for the cyclist is also conceivable.

\section{ACKNOWLEDGMENT}

The authors would like to thank the government of Lower Saxony, the Helmholtz foundation as well as the Volkswagen AG for funding the depicted research project CarS at the German Aerospace Center (DLR).

\section{REFERENCES}

[1] ETSI TR 102638 - Intelligent Transport Systems (ITS); Vehicular Communications; Basic Set of Applications; Definitions, V1.1.1 ed., European Telecommunications Standards Institute (ETSI), June 2009.

[2] simTD Deliverable D11.1 - Beschreibung der C2XFunktionen, V1.0 ed., Sicher Intelligente Mobilität Testfeld Deutschland, June 2009.

[3] T. Versteegh and B. Krosse, "From integrated urban awareness to safe cities for vulnerable road users," 2011.

[4] M. van Schijndel-de Nooij, S. d. Hair-Buijssen, R. Verweij, and V. Mathevon, "Savecap: Cyclist and pedestrian protection: From theory to practice," Lyon, June 2011.

[5] L. Andreone, F. Visintainer, and G. Wanielik, "Vulnerable road users thoroughly addressed in accident prevention: the watch-over european project," 2007.

[6] Intelligent Transport Systems (ITS); Vehicular Communications; C2C-CC Demonstrator 2008; Use Cases and Technical Specifications, V1.1.1 ed., European Telecommunications Standards Institute (ETSI), June 2008.

[7] T. Frankiewicz, A. Hinsberger, T. Lorenz, H.-J. Hilt et al., "Standortbestimmung und Integration von ITS Roadside Stations für die Anwendungsplattform Intelligente Mobilität," in AAET 2011. Automatisierungssysteme, Assistenzsysteme und eingebettete Systeme für Transportmittel, 2011.
[8] GRS-1: Dual Frequency RTK GNSS Receiver and Field Controller, Topcon Positioning Systems Inc., 2012.

[9] F. Köster, J. Gačnik, and M. Hannibal, "Serviceorientierung als Zugang zur Strukturierung von In-Vehicle Softwaresystemen," in 4. Braunschweiger Symposium: IMA 2008 Informationssysteme für mobile Anwendungen, Braunschweig, 2009.

[10] J. Gačnik, O. Häger, and M. Hannibal, "A serviceoriented architecture for the human centered design of intelligent transportation systems," in European Conference on Human Centered Design for Intelligent Transport Systems, Lyon, 2008.

[11] Global Positioning System Standard Ppositioning Service Performance Standard, 4th ed., Department of Defense United States of America, September 2008.

[12] Android Developers Reference, Google Inc., January 2012, URL: http://developer.android.com.

[13] M. Schröder, M. Hannibal, J. Gačnik, C. Harms, and T. Knostmann, "Ein Labor zur modellbasierten Gestaltung interaktiver Assistenz und Automation im Automotive-Umfeld," in AAET 2010. Automatisierungssysteme, Assistenzsysteme und eingebettete Systeme für Transportmittel, 2010. 